\title{
Cystoid macular edema
}

\author{
Tryfon G Rotsos' \\ Marilita M Moschos ${ }^{2}$ \\ 'Medical Retina Service, Moorfields \\ Eye Hospital, London, UK; \\ ${ }^{2}$ Department of Ophthalmology, \\ University of Athens, Greece
}

\begin{abstract}
We review the epidemiology, pathophysiology, and etiology of cystoid macular edema (CME). Inflammatory, diabetic, post-cataract, and macular edema due to age-related macular degeneration is described. The role of chronic inflammation and hypoxia and direct macular traction is evaluated in each case according to different views from the literature. The different diagnostic methods for evaluating the edema are described. Special attention is given to fluoroangiography and the most modern methods of macula examination, such as ocular coherence tomography and multifocal electroretinography. Finally, we discuss the treatment of cystoid macular edema in relation to its etiology. In this chapter we briefly refer to the therapeutic value of laser treatment especially in diabetic maculopathy or vitrectomy in some selected cases. Our paper is focused mainly on recent therapeutic treatment with intravitreal injection of triamcinolone acetonide and anti-VEGF factors like bevacizumab (Avastin), ranibizumab (Lucentis), pegaptamid (Macugen), and others. The goal of this paper is to review the current status of this treatment for macular edema due to diabetic maculopathy, central retinal vein occlusion and post-cataract surgery. For this reason the results of recent multicenter clinical trials are quoted, as also our experience on the use of intravitreal injections of anti-VEGF factors and we discuss its value in clinical practice.
\end{abstract}

Keywords: cystoid macular edema, anti-VEGF, fluoroangiography, OCT, multifocal electroretinography

\section{Introduction}

Cystoid macular edema (CME) represents a common pathologic sequel of the retina and occurs in a variety of pathological conditions such as intraocular inflammation, central or branch retinal vein occlusion, diabetic retinopathy and most commonly following cataract extraction. Histological studies show that radially orientated cystoid spaces consisting of ophthalmoscopically clear fluid are often clinically detectable in the macula area. These cysts seem to be areas of retina in which the cells have been displaced. In this review a brief synopsis is done about the pathophysiology and etiology of $\mathrm{CME}$, the different diagnostic methods for evaluating the CME are described and finally the therapeutic approaches of the disease are evaluated.

\section{Etiology: Pathogenesis Ocular inflammatory diseases CME in uveitis}

The exact pathogenesis of CME remains uncertain. CME develops when excess fluid accumulates within the macular retina. This is thought to occur following disruption of the blood-retinal barrier (BRB). Fluorescein angiography in the normal eye demonstrates the intact barrier well as the dye stays within blood vessels and does not leak into the retinal tissues. In particular, the avascular zone at the macula remains dark with no egress of dye. When the BRB is damaged, fluid accumulates within the retina both intra- and extracellularly (Yanoff et al 1984).

Extracellular fluid accumulation disturbs cell function and retinal architecture. Müller cells are thought to play an important role in acting as metabolic pumps which 
keep the macula dehydrated. However, intracellular fluid accumulation in the Müller cells may also occur in CME and further reduce macular retinal function. Vitreous traction may also play a part as demonstrated by the findings of Hirokawa and colleagues (1985) who showed that uveitic eyes with complete vitreous detachment tend to have fewer macular changes than those eyes without complete vitreous detachment. Hikichi and Trempe (1993) reported on 116 eyes with uveitis and demonstrated the importance of an attached vitreous: it was present in $78 \%$ of eyes with CME vs $22 \%$ of eyes without CME. In eyes with uveitis, damage to the integrity of the BRB results in leakage of dye during fluorescein angiography which accumulates in the macular area, often with a characteristic petalloid appearance.

Numerous different T-cell cytokines have been detected in both the intraocular fluids of inflamed eyes and the biopsies of involved ocular tissue and it is thought that cytokines such as interferon- $\gamma$, interleukin-2, interleukin-10, and tumor necrosis factor- $\alpha$ are key players in the generation of intraocular inflammation (Wakefield and Lloyd 1992). Other inflammatory mediators such as prostaglandins and chemokines are secreted by a variety of cell types involved in ocular inflammation and are also important inflammatory mediators in the eye. Although the initiating factors for most forms of uveitis in man are unknown, the onset of the inflammatory process in experimental models of uveitis is associated with the influx of T-cell lymphocytes, particularly of the CD4+ subtype (Lightman and Chan 1990).

In experimental models of uveitis, sensitive quantitative techniques have shown that the BRB integrity is breached at the same time that $\mathrm{T}$ cells enter the eye, suggesting that $\mathrm{T}$ cells are responsible for this breakdown (Lightman and Greenwood 1992). Whether one specific T cell-secreted cytokine is directly responsible for this is unknown and it is likely that many are capable of damaging the BRB. Data from experimental models also suggest that the BRB can be damaged to variable degrees and in a patchy fashion along a blood vessel wall (Lightman et al 1987). More severe damage in one area may allow large protein macromolecules into the retina, whereas in a nearby area less severe damage may allow in only low molecular weight solutes. These focal areas of BRB breakdown can seal up rapidly and other areas may then become involved. The exact mechanism allowing passage of fluid and molecules into the retina is unknown, but probably occurs via a transcellular route rather than through the tight junctions. As resolution of the inflammatory process occurs, cytokine production by the $\mathrm{T}$ cells is reduced, the BRB is no longer compromised, and abnormal fluid ingress into the retina stops. Good vision may be restored once any remaining intraretinal fluid is removed and the normal integrity of the BRB is restored. However, if the edema is chronic or very severe, permanent damage to the macular retinal cells may occur, with retinal thinning and fibrosis, such that resolution of the edema fluid may not result in return of normal vision (Guex-Crosier 1999). For example, eyes with bacterial or fungal endophthalmitis often have marked visual loss due to CME once the eye has been successfully treated. The over-whelming insult to the eye from infection and accompanying inflammation is likely to result in severe BRB damage and subsequent CME with significant, permanent visual loss.

\section{CME and pars planitis}

Macular edema and consequent loss of vision are the most frequent and serious complications of pars planitis (Henderly et al 1986). Persistent macular edema for more than 6 to 9 months leads to chronic macular changes, with permanent impairment of central vision; the degree of impairment reflects the severity of the changes. The presence of the pars plana exudates or membrane is more often, but not invariably, associated with more severe vitreous inflammation and CME (Henderly et al 1987).

\section{CME, HIV and immune recovery uveitis}

Although serous macular exudation has been described in patients with AIDS-related cytomegalovirus retinitis, CME is rarely encountered in this clinical setting (Cassoux et al 1999). However, the introduction of HAART has markedly changed the incidence and the prognosis of cytomegalovirus retinitis-related CME. In some patients the restoration of immune competence is associated with anterior segment and vitreous inflammatory reactions resulting in chronic vision threatening complications including CME (Cassoux et al 1999; Holland GN 1999; Kersten et al 1999). Other inflammatory conditions in which CME may occur include HLA-B27-associated acute anterior uveitis, sarcoidosis, birdshot retinochoroidopathy, Behcet's syndrome, toxoplasmosis, Eales' disease, idiopathic vitritis, VogtKoyanagi-Harada syndrome, and scleritis (Camras et al 1999; Dana et al 1996; Dodds et al 1999; Helm et al 1997; Schlaegel and Weber 1984).

\section{Postoperative cystoid macular edema}

Cystoid macular edema following cataract surgery was initially reported by Irvine in 1953 and is known as the Irvine-Gass syndrome (Irvine 1953). Approximately $20 \%$ of 
the patients who undergo uncomplicated phacoemulsification or extracapsular extraction develop angiographically proven CME (Peterson et al 1992; Jampol et al 1984). However, a clinically significant decrease in visual acuity is seen only in about $1 \%$ of these eyes (Bergman and Laatikainen 1994). If cataract extraction is complicated by posterior capsule rupture and vitreous loss, severe iris trauma or vitreous traction at the wound, there is a significantly higher incidence (up to $20 \%$ ) of clinically apparent CME, which is unrelated to the presence of AC-IOL (Bradford et al 1988). Clinically significant CME usually occurs within 3-12 weeks postoperatively, but in some instances its onset may be delayed for months or many years after surgery. Spontaneous resolution of the $\mathrm{CME}$ with subsequent visual improvement may occur within 3-12 months in $80 \%$ of the patients (Bonnet 1995). Cataract surgery in diabetic patients may result in a dramatic acceleration of pre-existing diabetic macular edema leading to poor functional visual outcome. This can be prevented provided the severity of the retinopathy is recognized preoperatively and treated appropriately with prompt laser photocoagulation either before surgery, if there is adequate fundal view, or shortly afterward (Flanagan 1993). Dowler and colleagues (1999), in a prospective clinical and angiographic study, reported that $69 \%$ of the eyes in which clinically significant macular edema arose in the first 6 months after cataract surgery showed spontaneous resolution of macular edema. In contrast it persisted in all eyes in which macular edema had been present at the time of surgery. Studies comparing phacoemulsification versus extracapsular cataract extraction in patients with diabetes revealed no difference in incidence of postoperative clinically significant macular edema between the two techniques emphasizing that early intervention when required is more critical to outcome than choice of surgical technique (Dowler et al 2000). Cystoid macular edema is one of the leading causes of poor postoperative visual acuity after cataract surgery in uveitis patients. Foster and colleagues (1992), in a retrospective study of uveitis patients undergoing extracapsular cataract extraction and posterior chamber intraocular lens implantation, reported $46 \%$ incidence of postoperative macular edema but in all cases improved or resolved with corticosteroid therapy. It has been suggested that the risk of macular edema is greater in uveitis patients with severe postoperative uveitis and preoperative anterior uveitis (Okhravi et al 1999).

The etiologic role of the vitreous in aphakic CME (ACME), initially described by Irvine (1953) as a complication of vitreous traction, was endorsed by Tolentino and Schepes (1965). Although the actual etiologic significance of vitreomacular traction has been questioned by Gass and Norton (1966), Reese and colleagues (1967) extended this hypothesis by speculating that, after cataract extraction, vitreous traction occurred following vitreous loss or delayed rupture of the anterior vitreous face. The hypothesis that vitreomacular traction causes $\mathrm{CME}$ has been confirmed histopathologically (Wolter 1981). Association between rupture of the anterior vitreous face and development of ACME has been proposed by several authors (Hitchings 1977; Irvine et al 1971). Other anterior segment changes, such as incarceration of the anterior vitreous to the corneal wound, have been related to ACME. This complication is associated not only with increased incidence of ACME but also with a worse functional prognosis (Federman et al 1980).

\section{Retinal vascular diseases Diabetic macular edema}

One of the most common causes of vision loss in patients with diabetes is diabetic macular edema (DME). The severity may range from mild and asymptomatic to profound loss of vision. DME is a general term defined as retinal thickening within two disc diameters of the foveal center; it can be either focal or diffuse in distribution. Focal edema is often associated with circinate rings of hard exudates (lipoprotein deposits) resulting from leakage from microaneurysms. Diffuse edema represents more extensive breakdown of the $\mathrm{BRB}$, with leakage from both microaneurysms and retinal capillaries. Cystic changes may appear within the macula, representing focal coalescence of exudative fluid. Clinically significant macular edema (CSME) is a form of DME that was precisely defined by the Early Treatment Diabetic Retinopathy Study (ETDRS) (Early Treatment Diabetic Retinopathy Study Research Group 1985, 1987). CSME exists if any of the following criteria are met:

- Any retinal thickening within $500 \mu \mathrm{m}$ of the foveal center.

- Hard exudates within $500 \mu \mathrm{m}$ of the foveal center that are associated with adjacent retinal thickening (which may lie more than $500 \mu \mathrm{m}$ from the foveal center).

- An area of retinal thickening at least 1 disc area in size, any part of which is located within 1 disc area of the foveal center.

The physics of diabetic macular edema are governed by a pair of hydrodynamic principles, Starling's law and LaPlace's law (Gardner et al 2002). Starling's law states that the net movement of fluid and molecules across the vessel wall is determined by the interplay between lumenal hydrostatic pressure, which drives fluid out of the vessel, and plasma colloid osmotic 
pressure, which draws fluid into the vessel. Lumenal hydrostatic pressure is often increased in diabetic eyes, due in part to coexisting systemic hypertension and in part to the increase in hydrostatic pressure that arises from focal retinal hypoxia. This favors the egress of fluid from vessels and increases the likelihood of developing macular edema. LaPlace's law states that a vessel will react to increased lumenal hydrostatic pressure by both dilating and becoming more tortuous. As a consequence, tight junctions between endothelial cells may become disrupted, again favoring fluid egress and macular edema.

\section{Retinal vein occlusion}

Retinal vein obstructions represent another common retinal vascular cause of CME. In patients with central retinal vein occlusion or a tributary branch occlusion involving the macula, CME is a major cause of visual loss. This edema, if severe or chronic ( $>8$ months), causes permanent diminution of vision secondary to disruption of the microscopic intraretinal connections and to the intracellular damage suffered by the visual elements (Coscas and Gaudric 1984). Persistent CME may be associated with vitreomacular attachment or hyperlipidemia and cardiovascular history, whereas it is inversely correlated to glaucoma (Glacet-Bernard et al 1996; Hikichi et al 1995). Finkelstein (1992) suggested that ischemic CME following branch retinal vein occlusion is often transient and, compared to perfused CME, has better prognosis for visual acuity. Another important sign of CME following obstructive venous retinopathy is the development of fluid blood levels in central cystoid spaces. Although fluid blood levels can occasionally be seen in diabetic, aphakic, or pseudophakic macular edema its occurrence is significantly more common in retinal vein occlusion. Therefore any diabetic patient displaying such a clinical finding should be suspected of having obstructive venous disease (Jones 1998).

\section{Diagnostic methods}

Slit-lamp examination with contact or noncontact lens makes it possible to detect retinal thickening, localized or extending to the posterior pole. The use of a narrow slit beam is useful in detecting cystoid spaces. Tests may be grouped into three categories according to whether one is analyzing the underlying pathogenesis, the effect of the macular edema on the retina, or its impact on visual function.

\section{Tests detecting disturbances in the blood-retinal barrier}

Macular edema may result from the breakdown of the BRB. This may occur at the level of the retinal pigment epithelium or the capillary endothelial cells. Various methods of investigation are utilized to detect disruption of the BRB in order to determine the presence and the extent of macular edema.

The fundus fluorescein angiogram is clinically the most widely available and useful test. It permits study of the circulation of the retina and choroid in normal and diseased states (Jaffe et al 1981). The amount of fluorescein leakage depends on the dysfunction of the retinal vascular endothelium. Although there is a significant correlation between visual acuity and the area covered by these cystoid changes, there is no relation between visual acuity and distance of cysts from the foveal avascular zone (Gass et al 1985). Fundus fluorescein angiography, apart from being a significant diagnostic modality also improves the accuracy of planning treatment for macular edema (Kylstra et al 1999). Late-phase stereoscopic pairs are useful in estimating the retinal thickness, the level of the leakage and the location and extent of cystic spaces.

\section{Tests detecting retinal tissue thickness}

Assessment of retinal thickness can be useful in the treatment and follow-up of macular edema. Retinal thickness at the posterior pole can be assessed by several methods. Because slit-lamp biomicroscopy and stereoscopic fundus photography are to some extent subjective, new imaging techniques for objective measurement of retinal thickness have been introduced to clinical use. The two most commonly used techniques are the OCT and the retinal thickness analyzer (RTA).

Optical coherence tomography is a noninvasive device that obtains cross-sectional, high-resolution images of the retina and thus may detect retinal thickening (Huang et al 1991). Microstructural features are determined by measuring the 'echo' time it takes for the light to reflect from the different structures at varying distances, analogous to A-scan ultrasonography. As the OCT operates with a near-infrared wavelength (about $840 \mathrm{~nm}$ ), the examination is of minimal discomfort for the patient (Ripandelli et al 1998). Optical coherence tomography examination is possibly indicated in the early detection and follow-up of patients with macular edema (Hee et al 1998). It has been shown to produce highly reproducible measurements and it is as effective at detecting macular edema as fluorescein angiography, but is superior at demonstrating axial distribution of the fluid (Hee et al 1998; Browning et al 2008). The RTA is a rapid screening instrument that generates a detailed map of retinal thickness (Zeimer et al 1989). Multiple cross sectional imaging generates a $3 \mathrm{D}$ reconstruction of the retina. The major advantage of the RTA is the option to scan a relatively wide area of 
the retina in a short acquisition time. It has been shown that RTA is a useful and sensitive tool, which provides objective measurement of the retinal thickness, facilitating the diagnosis and follow-up of diabetic macular edema, as well as evaluation of the efficacy of laser treatment in this condition (Zeimer et al 1989).

The scanning laser ophthalmoscope (SLO) has also been utilized in order to quantify retinal thickness by ophthalmoscopy and retinal topography (Oshima et al 1999) It is a rapid and noninvasive imaging method that provides quantitative analysis of macular cysts in addition to qualitative information not seen clinically (Mainster et al 1982). The chief advantage of the SLO is scanning a small focused spot to generate an image (rather than illuminating a large area), which provides a high contrast image. The infrared imaging of the SLO offers advantages over current imaging techniques by minimizing light scatter through cloudy media.

\section{Tests assessing retinal function}

Macular edema may potentially affect macular function as far as visual acuity and contrast sensitivity are concerned. Tests assessing macular function may be used indirectly to detect the effects of macular edema and follow up its treatment. Contrast sensitivity charts and electroretinography are both clinical and experimental tools. Contrast sensitivity has been documented as suffering specific changes in CME as well as other localized and generalized retinal disorders (Ghafour et al 1982). Ibanez and colleagues in a prospective comparative study evaluated the effect of pseudophakic CME on contrast sensitivity. They reported a statistically significant decrease in contrast sensitivity for patients who developed transient or persistent pseudophakic CME for all spatial frequencies studied at two months and for higher frequencies at 8.5 months following surgery, as opposed to no CME group. Reduction in contrast sensitivity may account for persistent difficulties experienced by patients despite good Snellen acuity (Ibanez et al 1993).

Electroretinography may also be utilized to follow up the treatment of macular edema. The focal electroretinogram (ERG) is the response evoked by the foveal cones of the retina to a brief flash of light focused on the fovea (Miyake et al 1993; Salzman et al 1986; Weiner et al 1997). The foveal ERG provides objective information on the presence or absence of organic disease at the macula. A very important development in the ERG-field in recent years is the multifocal-ERG recording system. This system allows assessment of ERG activity in small areas of retinal dysfunction. The multifocal-ERG introduced by Sutter and Tran (1992) allows the derivation simultaneously of
61 or 102 local ERG signals in a central visual field of about $60^{\circ}$ diameter around the fovea in a considerably short time of 4 to 8 minutes. So the decrease or retinal function due to regional disorders in the outer retinal layers can be described in details by this technique, which allows the functional mapping of the retina (Sutter and Tran 1992).

\section{Treatment Medical}

The challenge concerning the management of macular edema arises in the chronic and persistent case, for which a stepwise therapeutic approach is optimal. The clinician must always be alert to the possible side effects of the many effective, but potentially toxic, pharmaceutical agents used to treat this entity. Additionally, surgical management should be considered for unremitting cases of CME.

\section{Nonsteroidal antiinflammatory drugs}

In aphakic or pseudophakic CME, the occurrence of intraocular inflammation with synthesis of prostaglandins results in disruption of the tight junctions of the perifoveal retinal capillaries. Nonsteroidal anti-inflammatory drugs (NSAIDs) are useful as they inhibit the enzyme cyclooxygenase, which is required for the production of the prostaglandins as a degradation product of arachidonic acid. There is evidence that some NSAIDs may also act on other mediators. Experimental data showed that diclofenac at high concentrations inhibits the formation of lipoxygenase products (5-hydroxyeicosatetraenoic acid, leukotrienes) suggesting an additional regulatory role in the lipoxygenase pathway. However, the clinical relevance of the above property is still to be determined (Flach 2002). Topical NSAIDs, including ketorolac tromethamine $0.5 \%$, indomethacin $1 \%$, and diclofenac $1 \%$, are available and have been used either for treatment of macular edema following cataract surgery (Burnett et al 1983) or prophylactically to prevent angiographic edema (Jampol et al 1984). Although there is a multiplicity of studies addressing the issue of topical administration of NSAIDs for the prophylaxis or treatment of postoperative CME, the majority of them have not been randomized, or have had inadequate controls. In addition there is a diversity of final outcomes as some of the authors evaluate angiographic macular edema and others clinically significant CME, whereas most relevant outcomes such as visual acuity have been used only in a few studies.

\section{Carbonic anhydrase inhibitors}

Medical treatment of CME with carbonic anhydrase inhibitors (CAIs) has been known for over a decade (Cox et al 1988). 
Carbonic anhydrase inhibitors may alter the polarity of the ionic transport systems in the retinal pigment epithelium through the inhibition of carbonic anhydrase and $\gamma$-glutamyl transferase. As a result there is increased fluid transport across the retinal pigment epithelium from the sub-retinal space to the choroid with reduction of the edema. Carbonic anhydrase inhibitors have also been shown to have other direct effects both on retinal and retinal pigment epithelial cell function by inducing an acidification of the sub-retinal space, a decrease of the standing potential as well as an increase in retinal adhesiveness (Kita and Marmor 1992). Acetazolamide appears to be a particularly useful therapeutic agent in the management of macular edema due to retinitis pigmentosa (RP). A randomized crossover study reported improvement of visual acuity in more than $80 \%$ of all patients who received acetazolamide. The therapeutic effect may be independent of the reduction of macular edema, as judged by fluorescein angiography (Fishman et al 1989). A suggested treatment protocol includes a normal clinical starting dose of CAI of $500 \mathrm{mg}$ /day, which should be continued for at least 1 month to see an effect. The patients may reduce this dose over the course of therapy according to the subjective impression of treatment response.

\section{Steroids}

Steroids also inhibit the production of prostaglandins, but at a higher level in the biochemical pathway, by inhibiting the enzyme phospholipase A2, which catalyses the conversion of membrane lipids to arachidonic acid. By this process, steroids inhibit the formation of both prostaglandins and leukotrienes (Abe et al 1999). Locally their vasoconstrictive properties decrease intracellular and extracellular edema, suppress macrophage activity, and decrease lymphokine production. Corticosteroids may be administered topically, by periocular injection, orally and parenterally. Topical corticosteroids penetrate the corneal epithelium and reach the anterior chamber. The antiinflammatory properties of topical corticosteroids can be potentially helpful in treating CME caused by chronic iritis or iridocyclitis. Although the most common form of administration of systemic steroids is oral, there are published reports of series using highdose intravenous methylprednisolone for severe ocular inflammatory disease (Wakefield et al 1986) and for persistent pseudophakic CME with satisfactory results (Abe et al 1999). Intravitreal injection of triamcinolone acetonide has become a popular treatment, subsequently, a number of corticosteroidbased intravitreal implants have been developed to provide a sustained release of drug and make repeated intravitreal injections unnecessary. A promising treatment modality for patients poorly controlled or intolerant to repeated periocular corticosteroid injections, systemic corticosteroids, or steroid sparing immunosupressive agents has been suggested with the introduction of intraocular steroid-sustained drug delivery devices. It has been shown that these devices are nontoxic and produce constant intraocular drug levels for an extended period in human and experimental models (Hainsworth et al 1996; Jaffe et al 1996). There are currently four corticosteroidbased intravitreal implants under development. These include the dexamethasone biodegradable implant (Posurdex ${ }^{\circledR}$, Allergan, Irvine, CA), the helical triamcinolone acetonide implant (I-vation ${ }^{\mathrm{TM}}$ TA, SurModics, Eden Prairie, MN), the fluocinolone acetonide implant $\left(\right.$ Retisert $^{\circledR}$, Bausch and Lomb, Rochester, NY), and the fluocinolone acetonide - based implant that is injectable (Medidur ${ }^{\mathrm{TM}}$, pSivida, Boston, MA/Alimera Sciences, Alpharetta, GA). Triamcinolone acetonide has been reported to be effective in the management of macular edema (Jonas et al 2003; Loewenstein and Goldstein 2006). because it suppresses inflammation, reduces extravasation of fluid from leaking blood vessels, inhibits fibrovascular proliferation, and downregulates production of VEGF (Binz et al 2006). Triamcinolone can be administered by several routes, including intravitreal depot injection, periocular injection, posterior subtenon injection, and intravitreal implant. After depot injection, corticosteroid action peaks at 1 week, with residual activity persisting for 3 to 6 months. Intravitreal injection of triamcinolone is associated with significant adverse events, including elevated intraocular pressure in up to half of injected eyes (Jonas et al 2003) and cataract formation (Avitabile et al 2005; Loewenstein and Goldstein 2006), as well as injection-related complications such as endophthalmitis (Avitabile et al 2005; Loewenstein and Goldstein 2006) and retinal detachment (Androudi et al 2005). The efficacy of intravitreal use of triamcinolone acetonide was assessed by multifocal-ERG recording and OCT by Koutsandrea and colleagues (2007) and the results showed a real improvement of central retinal thickness and increase of the electrical activity of the foveal and perifoveal area 6 months after treatment (Figure 1). Periocular injections reduce the risk of serious complications such as endophthalmitis, but the duration of effect is shorter, and the therapeutic efficacy of triamcinolone administered by this route against DME is unclear.

\section{Surgical}

\section{Laser photocoagulation}

Photocoagulation is a therapeutic technique using a strong light source to coagulate tissue. Several theories attempted 

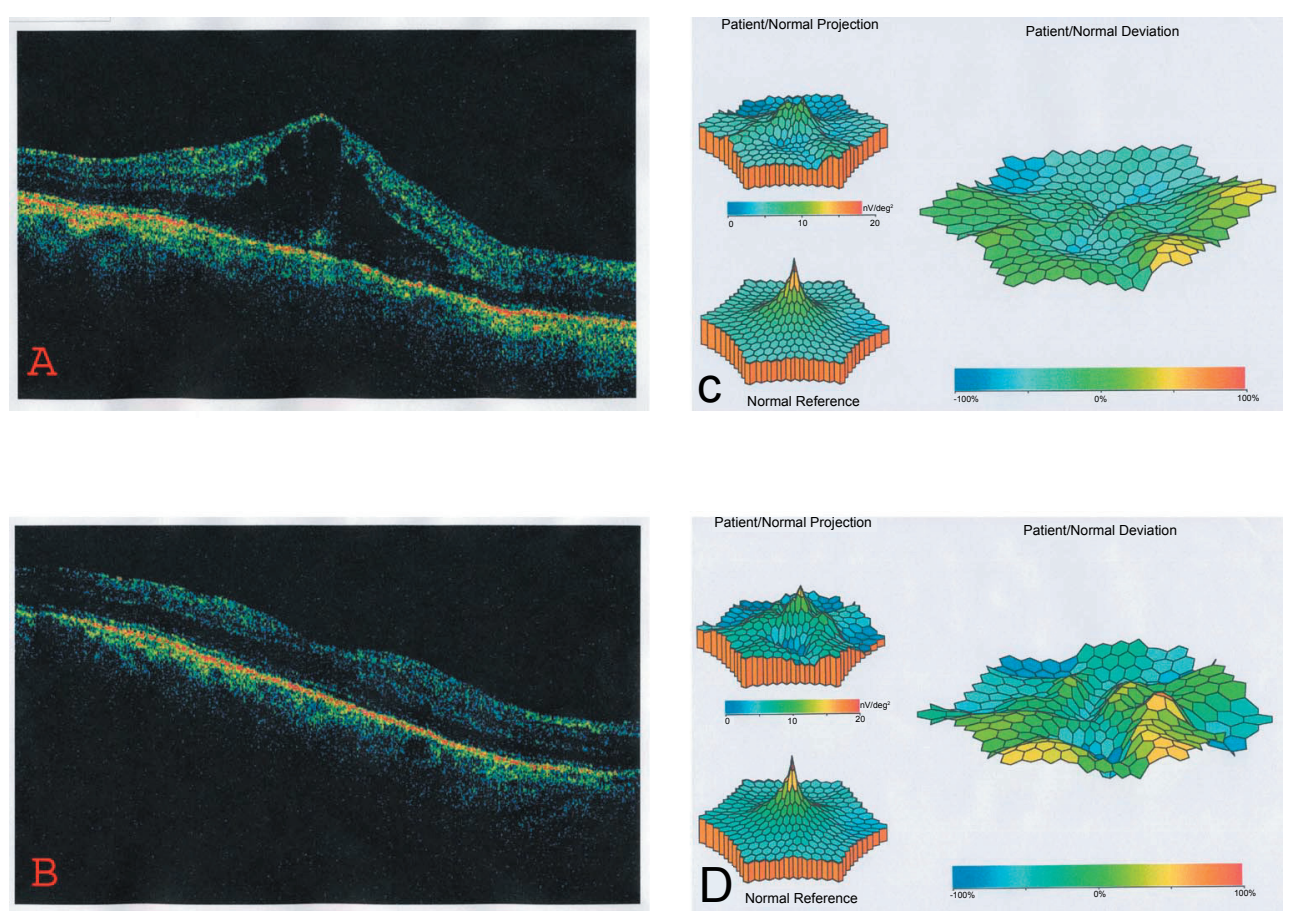

Figure I A 58-year-old man with pseudophakic CME. The pretreatment OCT shows an increase of the central retinal thickness (A), and the mf-ERG reveals a craterlike depression of the foveal and perifoveal area (B).Twelve months after treatment the OCT depicts the improvement of the central retinal thickness (C) and the mf-ERG shows a real recovery of the electrical activity of the foveal (area I) and parafoveal (area 2) area (D).

Abbreviations: CME, cystoid macular edema; mf-ERG, multifocal electroretinogram; OCT, optical coherence tomography.

to explain the beneficial effect of laser photocoagulation to macular edema. Laser lesions in experimental animals show a temporary breakdown of the BRB and a subsequent repair, as the retinal pigment epithelium cells adjacent to the burns proliferate and slide to replace the necrotic cells. The new retinal pigment epithelium cells produce tight junctions within several weeks, which restore the integrity of the retinal pigment epithelium barrier (Androudi et al 2005). An alternative hypothesis states that the grid laser by destroying photoreceptors reduces the oxygen consumption of the outer retina and allows oxygen to diffuse from the choroid to the inner retina, where it raises the oxygen tension and relieves hypoxia (Central Vein Occlusion Study Group 1995). This increased oxygen tension causes retinal arteriolar constriction and increased resistance in the arterioles, leading to reduced hydrostatic pressure in the capillaries and venules. The decreased hydrostatic pressure causes vessel constriction according to Laplace's law, vessel shortening, and less flux of fluid from vessel to tissue as is postulated in Starling's law (Molnar et al 1985). Diabetic macular edema and macular edema following branch retinal vein occlusion may improve following focal or grid laser photocoagulation. Laser treatment is not indicated for predominantly ischemic maculopathy. Most authors agree that the prognosis is best with localized leakage with or without circinate rings of hard exudates (The Branch Vein Occlusion Study Group 1984; The Early Treatment Diabetic Retinopathy Study Research Group 1987). Should PRP be also needed, this should be carried out after or in conjunction with macular treatment, because postPRP inflammation and altered retinal blood flow may result in deterioration of macular edema. Focal laser treatment aims to close or obliterate the microaneurysms producing focal areas of leakage (The Branch Vein Occlusion Study Group 1984). Grid photocoagulation may reduce leakage attributable to permeability abnormalities within dilated macular capillaries, with a positive effect on visual acuity and fluorescein leakage in diffuse diabetic macular edema, radiation retinopathy, and macular edema due to branch retinal vein occlusion. Side effects such as scotomas, corresponding to the laser burns, have been frequently noticed by the patients following photocoagulation. These scotomas generally fade over a period of several weeks. Symptoms are more likely to occur if laser burns are placed too close to each other.

\section{Vitrectomy}

\section{Diabetic macular edema}

Vitrectomy can be useful in eyes with DME if there is evidence of vitreomacular traction. There is a higher rate of posterior vitreous detachment in eyes without DME than in diabetic 
eyes with DME (Nasrallah et al 1988). In one published series, vitrectomy resulted in a $61 \%$ to $73 \%$ resolution of edema in DME cases without overt vitreoretinal interface abnormalities (Recchia et al 2005). Supplementing vitrectomy with the removal of the internal limiting membrane may improve outcomes, with one study showing that this approach provides better visual outcomes than a single session of modified grid laser in relieving persistent diffuse DME (Mason et al 2006). Vitrectomy is not without complications. Cataract formation is common, retinal detachments and recurrent vitreous hemorrhage may occur and intraocular pressure (IOP) may rise, leading to glaucoma.

\section{Aphakic/pseudophakic CME}

The Vitrectomy-Aphakic-Cystoid Macular Edema Study, a prospective, multicenter study of patients with chronic aphakic $\mathrm{CME}$, showed significant improvement in visual acuity following vitrectomy (Fung 1985). In eyes with vitreous loss at the time of cataract surgery and subsequent development of vitreous adhesions to anterior segment structures, lysis of vitreous strands to the cataract wound with the aid of Nd: YAG laser may reduce vitreous traction leading to resolution of CME (Katzen 1983).

\section{Future treatments}

Breakdown of the capillary BRB, causing macular edema, appears to be dependent on a number of active processes that may be open to pharmacological manipulation. In diabetes mellitus, hyperglycemia has been found to result in increased levels of dialglycerol, which in turn activates the protein kinase $\mathrm{C}$ (PKC) pathway. The latter functions as signal for growth factors, especially the vascular endothelial growth factor (VEGF), which plays a dominant role in retinal vascular leakage and formation of macular edema (Koya and King 1998). Experimental studies have clearly shown that blockage of VEGF action by inhibiting PKC $\beta$ may prevent the above process (Aiello et al 1995). Recently investigators have largely concentrated on LY333531, which is a highly selective inhibitor for the PKC $\beta$ isoform that has very little affect on other enzymes, minimizing the risk of systemic side effects resulting from its use. A randomized, double-masked, placebo-controlled study in diabetic patients of less than 10 years duration and no or mild retinopathy has reported normalization of retinal blood flow in patients treated with LY333531 as opposed to placebo-treated subjects (Aiello et al 1999).

\section{Anti-VEGF treatment}

Inhibition of VEGF has become a topic of interest in recent years in the area of age-related macular degeneration.
The properties of VEGF, and the consequences of its inhibition, also suggest a role for this approach in the management of DME.

In the pathophysiologic cascade leading to DME, chronic hyperglycemia leads to oxidative damage to endothelial cells as well as to an inflammatory response. The ensuing ischemia results in overexpression of a number of growth factors, including not only VEGF but also insulinlike growth factor-1, angiopoeitin-1 and -2, stromal-derived factor-1, fibroblast growth factor-2, and tumor necrosis factor (Grant et al 2004). Synergistically, these growth factors mediate angiogenesis, protease production, endothelial cell proliferation, migration, and tube formation. Tumor necrosis factor- $\alpha$ (TNF- $\alpha$ ) and VEGF play a role in the early stages of angiogenesis, with TNF- $\alpha$ promoting leukocyte adhesion and VEGF promoting leukostasis, resulting in ischemia.

Blockade of all involved growth factors will likely be necessary to completely suppress the detrimental effects of ischemia, but even isolated blockade of VEGF may have beneficial effects on DME. VEGF increases vascular permeability by relaxing endothelial cell junctions, which increases permeability and leakage. Inhibition of VEGF blocks this effect to some extent, as demonstrated in several recent clinical trials and case series involving the anti-VEGF molecules pegaptanib, ranibizumab, and bevacizumab. Pegaptanib sodium (Macugen ${ }^{\circledR}$, Eyetech Pharmaceuticals, Melville, NY/Pfizer, New York, NY) is an anti-VEGF aptamer, a small piece of RNA that selffolds into a shape that binds to and blocks the effects of VEGF $_{165}$, one isoform of the VEGF family of molecules. The drug is approved by the FDA for the treatment of age-related macular degeneration, and it has recently been studied in a phase II trial for DME (Cunningham et al 2005). In that study, 172 subjects with DME were randomized to receive a series of 3 intravitreal injections of pegaptanib (at entry and every 6 weeks) in 1 of 3 doses, or a sham injection, and were followed for 36 weeks. Additional injections or photocoagulation were permitted every 6 weeks through the end of the study. A total of $52 \%$ of patients in the $0.3-\mathrm{mg}$ and sham groups had baseline visual acuity of $<58$ letters; the remaining $48 \%$ had baseline visual acuity of $\geq 58$ letters. At the 36 -week mark, mean visual acuity had improved to $20 / 50$ in the pegaptanib 0.3-mg group (the dose that was approved by the FDA) versus only 20/63 in the sham group $(P=0.04)$. Mean central retinal thickness decreased by $68 \mathrm{mcm}$ in the $0.3-\mathrm{mg}$ group, whereas it increased by $4 \mathrm{mcm}$ in the 
sham group $(P=0.02)$. In addition, photocoagulation was required in $25 \%$ of the $0.3-\mathrm{mg}$ group compared with $48 \%$ of the sham group $(P=0.04)$. The injections were well tolerated, with a single case of endophthalmitis reported (1/652 injections, $0.15 \%)$.

Ranibizumab (Lucentis ${ }^{\mathrm{TM}}$, Genentech, San Francisco, $\mathrm{CA}$ ) is an antibody fragment that also binds and blocks the effects of VEGF. Unlike pegaptanib, ranibizumab binds and inhibits all isoforms of VEGF. Ranibizumab is also approved by the FDA for the treatment of age-related macular degeneration. A small, single-site, open-label trial was conducted in which 10 patients with DME were treated with a series of 3 monthly injections of 1 of 2 doses of ranibizumab ( 0.3 or $0.5 \mathrm{mg}$; the FDA approved the latter for macular degeneration) and then followed for 2 years (Chun et al 2006). Recently published data from this study indicate that at 3 months, 4 patients gained 15 or more letters of vision, 5 patients gained 10 or more letters, and 8 patients gained at least 1 letter. Mean central retinal thickness was reduced by $45 \mu \mathrm{m}$ in the $0.3-\mathrm{mg}$ group and by $198 \mu \mathrm{m}$ in the FDA-approved 0.5-mg group.

Bevacizumab (Avastin ${ }^{\circledR}$, Genentech, San Francisco, CA) is the full antibody from which ranibizumab is derived. This anti-VEGF molecule is FDA approved for systemic treatment of metastatic colon cancer, but not for any ophthalmic indications. Its use in conditions such as age-related macular degeneration, diabetic retinopathy, and DME is currently off-label.

A small, retrospective review of 16 eyes of 15 patients with macular edema secondary to central retinal vein occlusion recently reported the short-term anatomic and visual acuity outcomes after treatment with intravitreal bevacizumab (Iturralde et al 2006). Subjects received at least one injection of bevacizumab $1.25 \mathrm{mg}$, with repeat injections at 1 month at the investigator's discretion, with a mean of 2.8 injections per eye. One month after the initial injection, mean central macular thickness measured by OCT decreased from 887 to $372 \mu \mathrm{m}(P<0.001)$. Mean visual acuity improved from the pretreatment baseline of $20 / 600$ to $20 / 200$ at 1 month $(P=0.001)$ and to $20 / 138$ at 3 months $(P<0.001)$. In all, 14 of the 16 eyes demonstrated a halving of the visual angle, and no adverse events were noted. In a recent study, Moschos and Moschos (2008) showed that 3 months after the intravitreal use of bevacizumab, the electrical activity of the fovea and perifovea increased significantly (Figure 2, Figure 3). Anti-VEGF therapy for DME shows promise in preliminary studies. Larger studies are ongoing. VEGF
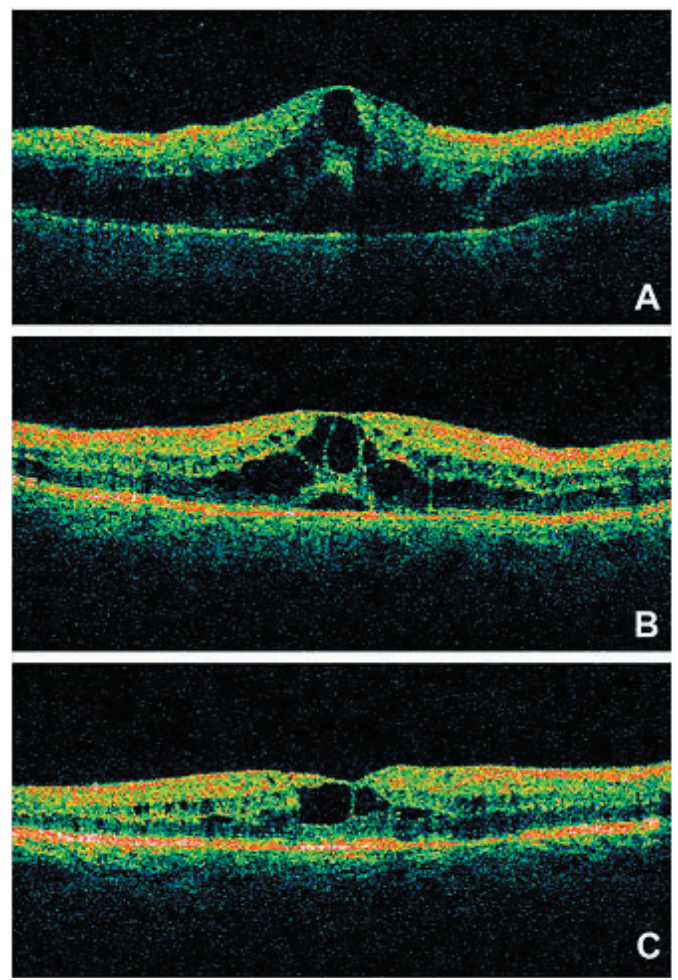

Figure 2 OCT recordings of the right eye of a 60 -year-old male patient at presentation (A), I month after treatment (B) and 3 months after treatment (C).The pretreatment OCT showed an increase of central retinal thickness. Three months after treatment the OCT depicted the decrease of the retinal thickness of the fovea. Abbreviation: OCT, optical coherence tomography.
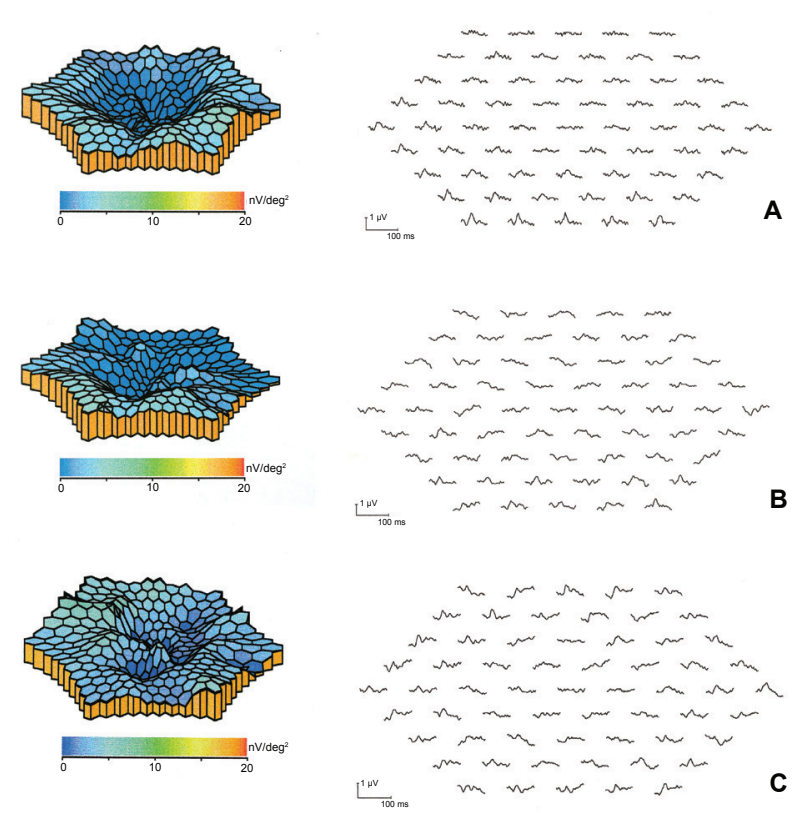

Figure 3 3D topographic plot and mf-ERG traces of the same eye at presentation (A), I month after treatment (B) and 3 months after treatment (C). Before treatment the mf-ERG revealed a craterlike depression of the foveal and perifoveal area. Three months after treatment the $\mathrm{mf}-\mathrm{ERG}$ showed an improvement of the electrical activity of the fovea (area I) and perifovea (area 2).

Abbreviations: 3D, three dimensional; mf-ERG, multifocal electroretinogram. 
inhibition may represent an important component of DME therapy in the future. Improvements in drug delivery will be necessary in order to avoid repeated intravitreal injections and the cumulative risk of endophthalmitis associated with this route of administration.

\section{Method of literature search}

In this review, we identified pertinent articles on macular edema from a combination of sources including electronic database searches and manual searches through the literature. A computerized search of the PubMed database (National Library of Medicine) was performed up to January 2008. The term 'macular edema' was used for a broad and sensitive search. Subsequently all the abstracts were carefully scanned and were divided into subcategories covering topics including 'pathogenesis', 'etiology', 'clinical manifestations', 'investigations and treatment of macular edema'. Non-English articles were included when deemed necessary. Copies of the entire articles were obtained. Reference lists of identified sources were used to glean more articles on the same topic. Additional books cited from these references were also used.

\section{Disclosure}

The authors report no conflicts of interest in this work.

\section{References}

Abe T, Hayasaka S, Nagaki Y, et al. 1999. Pseudophakic cystoid macular edema treated with high-dose intravenous methylprednisolone. $J$ Cataract Refract Surg, 25:1286-8.

Aiello LP, Pierce EA, Foley ED, et al. 1995. Suppression of retinal neovascularization in vivo by inhibition of vascular endothelial growth factor (VEGF) using soluble VEGF-receptor chimeric proteins. Proc Natl Acad Sci U S A, 92:10457-61.

Aiello LP, Bursell SE, Devris T et al. 1999. Protein kinase C beta selective inhibitor LY333531 ameliorates abnormal retinal hemodynamics in patients with diabetes. Diabetes, 48:A19.

Androudi S, Letko E, Meniconi M, et al. 2005. Safety and efficacy of intravitreal triamcinolone acetonide for uveitic macular edema. Ocul Immunol Inflamm, 13:205-12

Argon laser photocoagulation for macular edema in branch vein occlusion. 1984. The Branch Vein Occlusion Study Group. Am J Ophthalmol, 98:271-82.

Avitabile T, Longo A, Reibaldi A. et al. 2005. Intravitreal triamcinolone compared with macular laser grid photocoagulation for the treatment of cystoid macular edema. Am J Ophthalmol, 140:695-702.

Bergman M and Laatikainen L. 1994. Cystoid macular oedema after complicated cataract surgery and implantation of an anterior chamber lens. Acta Ophthalmol (Copenh), 72:178-80.

Binz N, Graham CE, Simpson K, et al. 2006. Long-term effect of therapeutic laser photocoagulation on gene expression in the eye. FASEB J, 20:383-5.

Bonnet S. 1995. [Repercussions of cataract surgery on the development of cystoid macular edema in the diabetic patient]. Bull Soc Belge Ophtalmol, 256:127-9.

Bradford JD, Wilkinson CP, Bradford RH, et al. 1988. Cystoid macular edema following extracapsular cataract extraction and posterior chamber intraocular lens implantation. Retina, 8:161-4.
Browning DJ, Glassman AR, Aiello LP, et al. 2008. Diabetic Retinopathy Clinical Research Network. Optical coherence tomography measurements and analysis methods in optical coherence tomography studies of diabetic macular edema. Ophthalmology, 115:1366-71.

Burnett J, Tessler H, Isenberg S, et al. 1983. Double-masked trial of fenoprofen sodium: treatment of chronic aphakic cystoid macular edema. Ophthalmic Surg, 14:150-2.

Camras CB, Fardeau C, Cassoux N, et al. 1999. [Ocular manifestations of Behçet's disease]. Ann Med Interne (Paris), 150:529-34.

Cassoux N, Lumbroso L, Bodaghi B, et al. 1999. Cystoid macular oedema and cytomegalovirus retinitis in patients with HIV disease treated with highly active antiretroviral therapy. Br J Ophthalmol, 83:47-9.

Central Vein Occlusion Study Group. 1995. Evaluation of grid pattern photocoagulation for macular edema in central vein occlusion. Central Vein Occlusion Study Group. report. Ophthalmology, 102:1425-33.

Chun DW, Heier JS, Topping TM, et al. 2006. A pilot study of multiple intravitreal injections of ranibizumab in patients with center-involving clinically significant diabetic macular edema. Ophthalmology, 113:1706-12.

Coscas G and Gaudric A. 1984. Natural course of nonaphakic cystoid macular edema. Surv Ophthalmol, 28(Suppl):471-84.

Cox SN, Hay E, Bird AC, et al. 1988. Treatment of chronic macular edema with acetazolamide, Arch Ophthalmol, 106:1190-5.

Cunningham ET Jr, Adamis AP, Altaweel M, et al. 2005. A phase II randomized double-masked trial of pegaptanib, an anti-vascular endothelial growth factor aptamer, for diabetic macular edema. Ophthalmology, 112:1747-57.

Dana MR, Merayo-Lloves J, Schaumberg DA, et al. 1996. Prognosticators for visual outcome in sarcoid uveitis. Ophthalmology, 103:1846-53.

Dodds EM, Lowder CY, Meisler DM. et al. 1999. Posterior segment inflammation in HLA-B27+ acute anterior uveitis: clinical characteristics. Ocul Immunol Inflamm, 7:85-92.

Dowler JG, Hykin PG, Hamilton AM, et al. 2000. Phacoemulsification versus extracapsular cataract extraction in patients with diabetes. Ophthalmology, 107:457-62.

Dowler JG, Sehmi KS, Hykin PG, et al. 1999. The natural history of macular edema after cataract surgery in diabetes. Ophthalmology, 106:663-8

Early Treatment Diabetic Retinopathy Study Research Group. 1985. Photocoagulation for diabetic macular edema. Early Treatment Diabetic Retinopathy Study report no 1. Arch Ophthalmol, 103:1796-806.

Early Treatment Diabetic Retinopathy Study Group. 1987. Techniques for scatter and local photocoagulation treatment of diabetic retinopathy: Early Treatment Diabetic Retinopathy Study Report no. 3. The Early Treatment Diabetic Retinopathy Study Research Group. Int Ophthalmol Clin, 27:254-64.

Early Treament Diabetic Retinopathy Study. 1991. Early photocoagulation for diabetic retinopathy. ETDRS report number 9. Early Treatment Diabetic Retinopathy Study. Research Group. Ophthalmology, 98(Suppl 5):766-85.

Federman JL, Annesley WH Jr, Sarin LK, et al. 1980. Vitrectomy and cystoid macular edema. Ophthalmology, 87:622-8.

Finkelstein D. 1992. Ischemic macular edema. Recognition and favourable natural history in branch vein occlusion. Arch Ophthalmol, 110:1427-34.

Fishman GA, Gilbert LD, Fiscella RG, et al. 1989. Acetazolamide for treatment of chronic macular edema in retinitis pigmentosa. Arch Ophthalmol, 107:1445-52.

Flach AJ. 2002. Topical nonsteroidal antinflammatory drugs in ophthalmology. Int Ophthalmol Clin, 42:1-11.

Flanagan DW. 1993. Current management of established diabetic eye disease. Eye, 7(Pt 2):302-8.

Foster RE, Lowder CY, Meisler DM, et al. 1992. Extracapsular cataract extraction and posterior chamber intraocular lens implantation in uveitis patients. Ophthalmology, 99:1234-41.

Fung WE. 1985. Vitrectomy for chronic aphakic cystoid macular edema. Results of a national, collaborative, prospective, randomized investigation. Ophthalmology, 92:1102-11. 
Gardner TW, Antonetti DA, Barber AJ, et al. 2002. Diabetic retinopathy: more than meets the eye. Surv Ophthalmol, 47(Suppl 2):S253-62.

Gass JD, Anderson DR, Davis EB, et al. 1985. A clinical, fluorescein angiographic, and electron microscopic correlation of cystoid macular edema. Am J Ophthalmol, 100:82-6.

Gass JDM and Norton EWD. 1966. Cystoid macular edema and papilledema following cataract extraction: A fluorescein fundoscopic and angiographic study. Arch Ophthalmol, 76:646-61.

Ghafour IM, Foulds WS, Allan D, et al. 1982. Contrast sensitivity in diabetic subjects with and without retinopathy. Br J Ophthalmol, 66:492-5.

Glacet-Bernard A, Coscas G, Chabanel A, et al. 1996. Prognostic factors for retinal vein occlusion: prospective study of 175 cases. Ophthalmology, 103:551-60.

Grant MB, Afzal A, Spoerri P, et al. 2004. The role of growth factors in the pathogenesis of diabetic retinopathy. Expert Opin Investig Drugs, 13:1275-93.

Guex-Crosier Y. 1999. The pathogenesis and clinical presentation of macular edema in inflammatory diseases. Doc Ophthalmol, 97:297-309.

Hainsworth DP, Pearson PA, Conklin JD, et al. 1996. Sustained release intravitreal dexamethasone. J Ocul Pharmacol Ther, 12:57-63.

Hee MR, Puliafito CA, Duker JS, et al. 1998. Topography of diabetic macular edema with optical coherence tomography. Ophthalmology, 105:360-70

Helm CJ, Holland GN, Webster RG, et al. 1997. Combination intravenous ceftazidime and aminoglycosides in the treatment of pseudomonal scleritis. Ophthalmology, 104:838-43.

Henderly DE, Genstler AJ, Rao NA, et al. 1986. Pars planitis. Trans Ophthalmol Soc UK, 105(Pt 2):227-32.

Henderly DE, Haymond RS, Rao NA, et al. 1987. The significance of the pars plana exudates in pars planitis. Am J Ophthalmol, 103:669-71.

Hikichi T and Trempe CL. 1993. Role of thevitreous in the prognosis of peripheral uveitis. Am J Ophthalmol, 116:401-5.

Hikichi T, Konno S, Trempe CL, et al. 1995. Role of the vitreous in central retinal vein occlusion, Retina, 15:29-33.

Hirokawa H, Takahashi M, Trempe CL, et al. 1985. Vitreous changes in peripheral uveitis. Arch Ophthalmol, 103:1704-7.

Hitchings RA. 1977. Aphakic macular oedema: A two-year follow-up study. Br J Ophthalmol, 61:628-30.

Holland GN. 1999. Immune recovery uveitis. Ocul Immunol Inflamm, 7:231-5.

Huang D, Swanson EA, Lin CP, et al. 1991. Optical coherence tomography. Science, 254:1178-81.

Ibanez HE, Lesher MP, Singerman LJ, et al. 1993. Prospective evaluation of the effect of pseudophakic cystoid macula edema on contrast sensitivity. Arch Ophthalmol, 111:1635-9.

Irvine AR, Bresky R, Crowder BM, et al. 1971. Macular edema after cataract extraction. Ann Ophthalmol, 3:1234-40.

Irvine AR. 1953. A newly defined vitreous syndrome following cataract surgery: interpreted according to recent concepts of the structure of the vitreous. Am J Ophthalmol, 36:599-619.

Irvine SR. 1953. A newly defined vitreous syndrome following cataract surgery, interpreted according to recent concepts of the structure of the vitreous. Am J Ophthalmol, 36:599-619.

Iturralde D, Spaide RF, Meyerle CB, et al. 2006. Intravitreal bevacizumab (Avastin) treatment of macular edema in central retinal vein occlusion: a short-term study. Retina, 26:279-84.

Jaffe GJ, Ben-Nun J, Guo H, et al. 2000. Fluocinolone acetonide sustained drug delivery device to treat severe uveitis. Ophthalmology, 107:2024-33.

Jaffe NS, Luscombe SM, Clayman HM, et al. 1981. A fluorescein angiographic study of cystoid macular edema. Am J Ophthalmol, 92:775-7.

Jampol LM, Sanders DR, Kraff MC, et al. 1984. Prophylaxis and therapy of aphakic cystoid macular edema. Surv Ophthalmol, 28(Suppl):535-9.

Jampol LM, Sanders DR, Kraff MC, et al. 1984. Prophylaxis and therapy of aphakic cystoid macular edema. Surv Ophthalmol, 28(Suppl):535-9.
Jonas JB, Kreissig I, Sofker A, et al. 2003. Intravitreal injection of triamcinolone for diffuse diabetic macular edema. Arch Ophthalmol, 121:57-61.

Jones WL. 1998. Fluid blood level in cystoid space resulting from central retinal vein occlusion. J Am Optom Assoc, 69:129-32.

Katzen LE, Fleischman JA, Trokel S, et al. 1983. YAG laser treatment of cystoid macular edema. Am J Ophthalmol, 95:589-92.

Kersten AJ, Althaus C, Best J, et al. 1999. Cystoid macular edema following immune recovery and treatment with cidofovir for cytomegalovirus retinitis. Graefes Arch Clin Exp Ophthalmol, 237:893-6.

Kita M and Marmor MF. 1992. Effects on retinal adhesive force in vivo of metabolically active agents in the subretinal space. Invest Ophthalmol Vis Sci, 33:1883-7.

Koutsandrea C, Moschos MM, Brouzas D, et al. 2007. Intraocular triamcinolone acetonide for pseudophakic cystoid macular edema: An OCT and mf-ERG study. Retina, 27:159-64.

Koya D and King GL. 1998. Protein kinase C activation and the development of diabetic complications. Diabetes, 47:859-66.

Kylstra JA, Brown JC, Jaffe GJ, et al. 1999. The importance of fluorescein angiography in planning laser treatment of diabetic macular edema. Ophthalmology, 106:2068-73.

Lightman S and Chan CC. 1990. Immune mechanisms in choroido-retinal inflammation in man. Eye, 4:345-53.

Lightman S and Greenwood J. 1992. Effect of lymphocytic infiltration on the blood-retinal barrier in experimental autoimmune uveoretinitis. Clin Exp Immunol, 88:473-7.

Lightman SL, Caspers-Velu LE, Hirose S, et al. 1987. Angiography with fluorescein-labeled dextrans in a primate model of uveitis. Arch Ophthalmol, 105:844-8.

Loewenstein A and Goldstein M. 2006. Intravitreal triamcinolone acetonide for diabetic macula edema. Isr Med Assoc J, 8:426-7.

Mainster MA, Timberlake GT, Webb RH, et al. 1982. Scanning laser ophthalmoscopy. Clinical applications. Ophthalmology, 89:852-7.

Mason JO III, Colagross CT, Vail R, et al. 2006. Diabetic vitrectomy: risks, prognosis, future trends. Curr Opin Ophthalmol, 17:281-5.

Miyake Y, Miyake K, Shiroyama N. et al. 1993. Classification of aphakic cystoid macular edema with focal macular electroretinograms. Am J Ophthalmol, 116:576-83.

Molnar I, Poitry S, Tsacopoulos M, et al. 1985. Effect of laser photocoagulation on oxygenation of the retina in miniature pigs. Invest Ophthalmol Vis Sci, 26:1410-4.

Moschos MM and Moschos MN. 2008. Intraocular bevacizumab for macular edema due to CRVO. A multlifocal-ERG and OCT study. Doc Ophthalmol, 116:147-52.

Nasrallah FP, Jalkh AE, Van Coppenolle F, et al. 1988. The role of the vitreous in diabetic macular edema. Ophthalmol, 95:1335-9.

Okhravi N, Lightman SL, Towler HM, et al. 1999. Assessment of visual outcome after cataract surgery in patients with uveitis. Ophthalmology, 106:710-22.

Oshima Y, Emi K, Yamanishi S, et al. 1999. Quantitative assessment of macular thickness in normal subjects and patients with diabetic retinopathy by scanning retinal thickness analyzer. Br J Ophthalmol, 83:54-61.

Peterson M, Yoshizumi MO, Hepler R, et al. 1992. Topical indomethacin in the treatment of chronic cystoid macular edema. Graefes Arch Clin Exp Ophthalmol, 230:401-5.

Recchia FM, Ruby AJ, Carvalho-Recchia CA, et al. 2005. Pars plana vitrectomy with removal of the internal limiting membrane in the treatment of persistent diabetic macular edema. Am J Ophthalmol, 139:447-54.

Reese AB, Jones IS, Cooper WC, et al. 1967. Macular changes secondary to vitreous traction. Am J Opthtalmol, 64:544-9.

Ripandelli G, Coppé AM, Capaldo A, et al. 1998. Optical coherence tomography. Semin Ophthalmol, 13:199-202.

Salzman J, Seiple W, Carr R, et al. 1986. Electrophysiological assessment of aphakic cystoid macular oedema. Br J Ophthalmol, 70:819-24.

Schlaegel TF and Weber JC. 1984. The macula in ocular toxoplasmosis. Arch Ophthalmol, 102:697-8. 
Sutter EG and Tran D. 1992. The field topography of ERG component in man. The photopic luminance response. Vision Res, 32:433-66.

Tolentino FI and Schepens CL. 1965; Edema of posterior pole after cataract extraction: A biomicroscopic study. Arch Ophthalmol, 74:781-6.

Wakefield D, McCluskey P, Penny R. et al 1986. Intravenous pulse methylprednisolone therapy in severe inflammatory eye disease. Arch Ophthalmol, 104:847-51.

Wakefield D and Lloyd A. 1992. The role of cytokines in the pathogenesis of inflammatory eye disease. Cytokine, 4:1-5.
Weiner A, Christopoulos VA, Gussler CH, et al. 1997. Foveal cone function in nonproliferative diabetic retinopathy and macular edema. Invest Ophthalmol Vis Sci, 38:1443-9.

Wolter JR. 1981. Cystoid Macular edema in vitreo-retinal traction. Ophthalmic Surg, 12:900-4.

Yanoff M, Fine BS, Brucker AJ, et al. 1984. Pathology of humancystoid macular edema. Surv Ophthalmol, 28(Suppl):505-11.

Zeimer RC, Shahidi M, Mori MT, et al. 1989. In vivo evaluation of a noninvasive method to measure the retinal thickness in primates. Arch Ophthalmol, 107:1006-9. 